\title{
O QUE SE PESQUISA EM ENSINO CONTÁBIL? ANÁLISE DO CONGRESSO USP DE CONTABILIDADE E INICIAÇÃO CIENTÍFICA
}

\author{
WHAT IS RESEARCHED IN ACCOUNTING EDUCATION? \\ ANALYSIS OF THE USP CONFERENCE ON ACCOUNTING AND INTRODUCTION TO \\ SCIENTIFIC RESEARCH
}

\author{
ELISABETH DE OLIVEIRA VENDRAMIN \\ Universidade Federal do Mato Grosso do Sul. Endereço: Rua, Senador \\ Filinto Müller, 1015 | Cidade Universitária | 79046-460 | Mato Grosso do \\ Sul/MS | Brasil. \\ Dhttp://orcid.org/0000-0002-9082-2256 \\ beth.vendramin@gmail.com
}

\author{
JOÃO PAULO RESENDE DE LIMA \\ Universidade de São Paulo. Endereço: Avenida Professor Luciano \\ Gualberto, 908 | Butantã | 05508-010 | São Paulo/SP | Brasil. \\ (1) https://orcid.org/0000-0002-4703-2603/ \\ jp.resendelima@hotmail.com
}

\section{ISADORA JEANE DOS SANTOS}

Universidade Federal do Mato Grosso do Sul. Endereço: Rua, Senador Filinto Müller, 1015 | Cidade Universitária | 79046-460 | Mato Grosso do Sul/MS | Brasil.

D http://orcid.org/0000-0001-5313-5150

isadora.jeane2001@gmail.com

\section{MARCUS VINICIUS PEREIRA GUASSO}

Universidade Federal do Mato Grosso do Sul. Endereço: Rua, Senador Filinto Müller, 1015 | Cidade Universitária | 79046-460 | Mato Grosso do Sul/MS | Brasil.

(D) http://orcid.org/0000-0003-4852-2396

mguasso@outlook.com

\section{NATÁLIA FERNANDES}

Universidade Federal do Mato Grosso do Sul. Endereço: Rua, Senador Filinto Müller, 1015 | Cidade Universitária | 79046-460 | Mato Grosso do Sul/MS | Brasil.

Dhttp://orcid.org/0000-0002-3139-743X

fernandes.nataly@gmail.com

\section{RESUMO}

O presente trabalho tem como objetivo analisar o campo de estudos sobre ensino e pesquisa contábil do USP International Conference in Accounting e do Congresso USP de Iniciação Científica em Contabilidade. Para tal, foi realizada uma pesquisa bibliométrica com obtenção dos dados por meio de consulta aos anais dos congressos. Ao total, foram analisados 209 artigos relativos ao Congresso Internacional e 126 artigos referente ao Congresso de Iniciação 
Científica. Os dados apontam que a participação de artigos aceitos ficou entre 1,85\% e 34,15\%, com média de $8,11 \%$ para o Congresso Internacional e $15,38 \%$ para o Congresso de Iniciação Científica. 80,6\% dos artigos de ambos os congressos possuem entre 2 e 4 autores, sendo que a maior rede de coautorias está no Congresso de Iniciação Científica. Com relação ao sexo, a autoria está equilibrada quando o olhar se volta para os dois eventos. Entretanto, as mulheres têm maior participação no Congresso de Iniciação Científica e os homens maior participação no Congresso Internacional. Diante desses resultados, conclui-se que a área de ensino e pesquisa constitui um campo periférico nos dois congressos devido a sua baixa quantidade de artigos em relação ao total, além do fato de que essas conferências possuem normas sociais distintas em relação a autoria. Assim, o presente artigo contribui para a literatura ao apresentar e discutir aspectos relacionados a constituição do campo científico de Ensino e Pesquisa em Contabilidade, compondo um perfil analítico da linha de ensino e pesquisa em um dos principais eventos da área.

Palavras-chave: Educação Contábil. Pesquisa Contábil. Campo Científico. Levantamento Bibliográfico. Análise de Conteúdo.

\begin{abstract}
This work aims to analyze the field of studies on teaching and accounting research at the USP International Conference in Accounting and the USP Conference of Introduction to Scientific Research in Accounting. For this purpose, bibliometric research was carried out, with data obtained by consulting the Conference proceedings. A total of 209 papers related to the International Conference and 126 papers related to the Conference of Introduction to Scientific Research were analyzed. The data indicate that accepted papers' participation was between $1.85 \%$ and $34.15 \%$, with an average of $8.11 \%$ for the International Conference and $15.38 \%$ for the Conference of Introduction to Scientific Research. $80.6 \%$ of the papers of both Conferences have between 2 and 4 authors, and the largest network of partnerships is in the Conference of Introduction to Scientific Research. Regarding gender, the authorship is balanced when looking at the two Conferences; however, women have greater participation in the Conference of Introduction to Scientific Research, and men have greater participation in the International Conference. Given these results, we concluded that the area of teaching and research constitutes a peripheral area in the two Conferences due to the low number of articles concerning the total, besides the fact that the Conferences have different social norms about authorship. Thus, this article contributes to the literature by presenting and discussing aspects related to the constitution of the scientific field of Teaching and Research in Accounting, composing an analytical profile of the teaching and research line in one of the leading conferences in the area.
\end{abstract}

Keywords: Accounting Education. Accounting Research. Scientific Field. Bibliographic Research. Content Analysis.

\title{
1 INTRODUÇÃO
}

A pós-graduação em Contabilidade no Brasil teve seu início na década de 1970 com a abertura dos cursos de mestrado e doutorado da Faculdade de Economia, Administração e Contabilidade da Universidade de São Paulo (FEA/USP), marco que pode ser visto como o princípio da pesquisa acadêmica contábil no Brasil (Peleias, Silva, Segreti, \& Chirotto, 2007). Desde então, a pesquisa em Contabilidade no país tem evoluído ao agregar novos temas e metodologias. Diante dessa evolução, observa-se um delineamento mais claro de um campo científico marcado por diferentes escolas de pensamento compostas por estruturas, relações e disputas de poder (Bourdieu, 1983; Kuhn, 1978; Costa \& Martins, 2017). 
A partir do desenvolvimento das escolas de pensamento e das disputas de poder do campo científico, a comunidade contábil vai se autorregulando por meio da vigilância epistemológica (Menafra, 2007) e das lutas pelo monopólio da competência científica (Bourdieu, 1983) ao definir tópicos e métodos que são mais importantes para o desenvolvimento da área e tópicos que não devem ser pesquisados ou deixados à margem (Homero Junior, 2017). Na comunidade brasileira de Contabilidade, era possível observar que dentre tais tópicos deixados à margem e que enfrentam pré-conceitos acadêmicos por meio de perguntas como "isso é pesquisa Contábil por acaso?" (Ganz, Lima, \& Haveroth, 2019) encontram-se aqueles relacionados ao ensino e pesquisa. Contudo, tal cenário tem se alterado no decorrer do tempo e a tal linha de pesquisa tem se consolidado no cenário nacional e internacional (Vendramin \& Araujo, 2016).

A razão para as temáticas relacionadas ao ensino e à pesquisa em Contabilidade terem sido relegadas à margem no Brasil pode ser decorrência da baixa autonomia do campo científico em relação ao campo profissional. Assim, temas diretamente não relacionados à profissão contábil acabam sofrendo resistência da área (Homero Junior, 2017) além de terem a qualidade de suas pesquisas questionada (Tharapos \& Marriott, 2020). Evidências da marginalização desse ramo é o fato de este existir, formalmente, em apenas um dos programas de pós-graduação do Brasil segundo pesquisa feita com dados de até 2014 (Vendramin, 2014), além de figurar de maneira mais presente em periódicos de avaliação mais baixa (Homero Junior \& Said, 2018). Contudo, observa-se que, dentro da comunidade científica contábil brasileira, iniciou-se um processo de construção de um grupo voltado para as pesquisas relacionadas ao ensino e pesquisa em Contabilidade (Vendramin \& Araujo, 2016).

As temáticas estudadas nessa linha - especificamente na questão do ensino - perpassam temas relacionados ao corpo docente (Miranda, Santos, Casa Nova, \& Cornacchione Junior, 2013; Lima \& Araújo, 2019), corpo discente (Santos, 2001; Vendramin, 2018), Instituições de Ensino Superior (Apostolou, Dorminey, Hassell, \& Rebele, 2018), currículo (Guimarães, Slomski, \& Gomes, 2010), metodologias de ensino e aprendizagem (Soares, Bulaon, Casa Nova, \& Picolli, 2019; Nagib \& Silva, 2020; Cruz, Miranda, \& Leal, 2020), dentre outros. Na vertente de pesquisa, observam-se trabalhos de cunho epistemológico que propõe (re)pensar a construção do conhecimento e da pesquisa contábil, de estudos acerca de métodos e metodologias de pesquisa (Martins, 2012; Homero Junior, 2017; Ganz et al., 2019) e de indicadores de produtividade (Soares, Lima Filho e Casa Nova, 2020).

Além da construção de comunidades, uma parte importante para a construção do conhecimento é sua comunicação e citação em congressos e periódicos, visto que a partir dessa divulgação o conhecimento passa a ser "validado" e aceito pela comunidade (Latour, 1987; Fogarty \& Liao, 2009). Diante dessa importância, faz-se necessário avaliar como o meio de ensino e pesquisa tem se comportado dentro da comunidade científica e como os principais meios de comunicação da pesquisa científica em Contabilidade tem aceitado ou não artigos relacionados à tal temática.

Assim sendo, o presente trabalho tem como objetivo analisar a esfera de estudos sobre ensino e pesquisa contábil do USP International Conference in Accounting e do Congresso USP de Iniciação Científica em Contabilidade. Para tal, adotamos como conceito de campo o proposto por Bourdieu (1983) e, por meio de indicadores bibliométricos, destacamos os principais agentes dessa área, além da delimitação de temáticas e metodologias adotadas pelo setor e seus agentes. O estudo justifica-se pela necessidade de repensar a pesquisa contábil nas diferentes áreas financeira, gerencial, mercados e educação e pesquisa - visto que existem trabalhos que indicam uma possível estagnação desse campo (Moser, 2012; Rebele \& St. Pierre, 2015; Lima, Serrano, \& Ferreira, 2020) de forma que a pesquisa com caráter epistemológico - ou seja, a pesquisa sobre a pesquisa - ganha ainda mais importância. O trabalho se apoia ainda com base no fato de que a sociedade tem passado por mudanças sociais e estruturais que têm transformado as dinâmicas de ensino e aprendizagem, trazendo a necessidade de refletir sobre a pesquisa nessa 
esfera. Assim, discutir a produção científica acerca dessas temáticas pode auxiliar a melhor compreender o campo visando diagnosticar possíveis problemas.

Para alcançar o objetivo proposto, foi realizada uma pesquisa documental nos anais do USP International Conference in Accounting e Congresso USP de Iniciação Científica em Contabilidade no período de 2000 a 2019. Tal levantamento resultou em 335 artigos que foram classificados em (i) Rendimento Acadêmico; (ii) Currículo; (iii) Docência; (iv) Evasão; (v) História da Contabilidade; (vi) Levantamento Bibliográfico; (vii) Metodologias de Ensino e Aprendizagem; (viii) Corpo Discente; (ix) Perfil Profissional; (x) Pesquisa Científica; (xi) Outros; e posteriormente tiveram fatores bibliométricos analisados.

Os resultados da pesquisa indicam que a área de Educação é incipiente nos congressos analisados, com características diferentes de rede de autoria, filiação informada pelos autores e sexo, além de existir diferenças encontradas entre o USP International Conference in Accounting e o Congresso de Iniciação Científica. Diante disso, o presente artigo contribui para a literatura ao apresentar um perfil analítico da linha de ensino e pesquisa em Contabilidade em um dos principais congresso da área contábil. O trabalho também discute a constituição de um campo da pesquisa em Contabilidade e apresenta alguns de seus delineamentos sociais como principais agentes e agentes institucionais, assim como os temas mais estudados e emergentes. Para a prática, ao apresentar possíveis lacunas de pesquisa, o presente trabalho contribui para pesquisadores e pesquisadoras que desejam ingressar na área de ensino e pesquisa.

O texto estrutura-se em cinco seções, sendo a primeira essa introdução. Em seguida, são discutidos procedimentos da construção social de conhecimentos, assim como a importância do conhecimento construído pelo campo de ensino e pesquisa em Contabilidade. Posteriormente, apresentam-se os procedimentos metodológicos aplicados à pesquisa. Por fim, são analisados e discutidos os resultados da pesquisa, assim como são apresentadas as considerações finais do estudo.

\section{REFERENCIAL TEÓRICO}

O processo de construção do conhecimento científico pode ser analisado por diferentes correntes epistemológicas e adotar diferentes contornos para cada uma. Aqui, considera-se que a construção do conhecimento é um processo social que envolve diferentes agentes e que é histórico-socialmente contextualizado (Bourdieu, 1983; Costa \& Martins, 2017). A partir dessa visão social do conhecimento, é importante entender o papel que os agentes ocupam nesse processo.

Para Kuhn (1978), um dos principais elementos para a construção do conhecimento é a noção de paradigma. Segundo o autor, paradigmas são "as realizações científicas universalmente reconhecidas que, durante algum tempo, fornecem problemas e soluções modelares para uma comunidade de praticantes de uma ciência" (p. 13). Dessa forma, observa-se que o paradigma une uma comunidade científica e, de acordo com Kuhn, aqueles que não adotam tal modelo caem no ostracismo.

Essa noção tem sido amplamente aplicada às ciências naturais, contudo é muito criticada quando empregada às ciências sociais de maneira geral (Caniato, 2005). Para as ciências sociais aplicadas, a discussão acerca dos paradigmas de pesquisa tem se pautado, principalmente, na discussão apresentada por Burrell e Morgan (1979) que organiza pressupostos metateóricos em quatro conjuntos de paradigmas. Esses, propostos por Burrell e Morgan (1979), "possuem caráter rival e excludentes, e focam as diferenciações ontológicas e epistemológicas" (Sauerbronn, Ayres, \& Lourenço, 2017).

Além do caráter rival entre os paradigmas, é importante reconhecer a existência de diferentes escolas de pensamento dentro de um mesmo modelo e que também adotam caráter competitivo (Morgan, 1980). A partir disso, percebe-se que a constituição de uma comunidade científica perpassa pelo processo de estabelecimento de regras e normas - formais e informais - 
que dão contorno à comunidade e ao que se pesquisa nessa comunidade. Nesse sentido, Bourdieu (1983, p. 1) afirma que:

[o] campo científico [...] é o lugar, o espaço de jogo de uma luta concorrencial. O que está em jogo especificamente nessa luta é o monopólio da autoridade científica definida, de maneira inseparável, como capacidade técnica e poder social; ou, se quisermos, o monopólio da competência científica, compreendida enquanto capacidade de falar e de agir legitimamente (isto é, de maneira autorizada e com autoridade), que é socialmente outorgada a um agente determinado.

Tem-se, dessa maneira, que as comunidades científicas podem ser vistas como um conjunto de agentes que buscam se legitimar no campo para definir o que possui caráter científico ou não. Além da comunidade científica e seus conflitos internos por poder, outro aspecto importante no processo de construção do conhecimento, e que perpassa pelos jogos de poder, é o que esse grupo aceita ou não. O processo de aceitação por uma comunidade científica costuma se dar por meio das publicações dos artigos em congressos e periódicos (Oliveira, 2002).

Comunicar os achados do processo de pesquisa científica é uma etapa importante do processo científico, visto que, como aponta Latour (1987, p. 70), "há algo ainda pior do que ser criticado ou demolido por leitores descuidados: é ser ignorado". De acordo com o autor, para a construção de um fato científico, é preciso que as produções sejam lidas e citadas para ganharem notoriedade e legitimidade dentro de sua comunidade.

Segundo Oliveira (2002), a comunicação dos resultados de uma pesquisa é o momento em que o meio acadêmico e a sociedade em geral passam a ter acesso ao conhecimento científico construído. Diversos são os meios de divulgação, entretanto, para um artigo científico, o caminho mais comum é que o(s) autor(es) submetam o artigo para apresentação em um evento científico, no qual existe a possibilidade de receber a contribuição de uma plateia que possui interesse no tema. Após a exposição em congresso, o(s) autor(es) tem a oportunidade de fazer melhorias no artigo - se julgarem necessárias - e então submeter a um periódico. Esse é o caminho geralmente seguido por um artigo científico no que tange ao processo de divulgação.

Os congressos científicos e periódicos, ligados à área contábil, têm abertura para o envio de artigos ligados a temas como contabilidade financeira, contabilidade e controle gerencial, finanças, educação contábil e pesquisa. Entretanto, o número de artigos aceitos não é proporcional entre os temas. Um exemplo ilustrativo é um levantamento feito por Vendramin (2014) entre os artigos aceitos no Congresso USP e Anpcont, nos anos de 2010 a 2013, que demonstra que $36 \%$ dos artigos estão situados na área de Contabilidade para usuários externos, $25 \%$ na área de Controladoria e Contabilidade Gerencial e $15 \%$ se divide entre Educação Contábil e Pesquisa Contábil.

Tal levantamento sugere o delineamento das temáticas dominantes nos congressos da área - ou, como denomina Chua (1986), o mainstream da área. A partir disso, observa-se que os estudos de ensino e pesquisa caracterizam a periferia do campo de Contabilidade, podendo seu caráter científico ser contestado pelo mainstream. A partir desse contexto, em seguida é apresentada a discussão acerca da importância das pesquisas em ensino e pesquisa contábil.

\subsection{Importância das pesquisas em Ensino Contábil}

A contabilidade como ciência social evoluiu com o intuito de atender às necessidades dos diferentes usuários, uma vez que "com o crescimento da dimensão e da complexidade das atividades empresariais, as necessidades de informações por parte dos administradores e dos demais usuários da Contabilidade tornam-se cada vez mais distintas" (Paulo \& Martins, 2007, p. 2). A evolução para atender as necessidades informacionais parte do princípio de que "o objetivo 
básico da contabilidade, [...] pode ser resumido no fornecimento de informações econômicas para vários usuários, de forma que propiciem decisões racionais” (Iudícibus, 2004, p. 25).

Por ser tanto o objeto de estudo da contabilidade (Martins \& Carvalho, 2011) quanto seu produto final, a informação contábil, seu impacto nos mercados e qualidade foram foco de diversas pesquisas em âmbito nacional e internacional. No contexto da importância da informação contábil, justificam-se os estudos de educação nessa área, visto que buscam melhorar a qualificação pedagógica dos docentes e o aprimoramento do ensino e, consequentemente, a formação dos profissionais de contabilidade (Miranda et al., 2013). Além disso, segundo Tharapos \& Marriott (2020), a esfera de ensino em Contabilidade possui potencial para contribuir diretamente para o avanço da profissão por lidar com as rápidas e constantes mudanças do mundo do trabalho.

Estudos que focam a formação do contador se fazem necessários, pois "num sistema contábil os eventos econômicos são as fontes básicas da informação contábil e o contador atua como transmissor, observando esses eventos e codificando-os para transmitir a informação por meio dos relatórios contábeis" (Stroeher \& Freitas, 2008, p. 5). Pressupõe-se uma relação positiva entre a qualificação do contador e a qualidade da informação contábil, visto que segundo a Teoria do Capital Humano "a aquisição de mais conhecimentos e habilidades aumenta o valor do capital humano das pessoas, aumentando sua empregabilidade, produtividade e rendimento potencial" (Martins \& Monte, 2009, p. 4).

A educação contábil é formada por subsistemas como associações de profissionais, empresas, instituições públicas, órgãos regulamentadores e instituições de ensino (Riccio \& Sakata, 2004), assim, todos esses subsistemas afetam a formação do contador de maneira direta ou indireta. Laffin (2002) acrescenta à discussão o papel desempenhado pelo currículo e pelo professor na formação do profissional da área. A formação do contador ainda é influenciada pela Instituição de Ensino Superior (IES), já que é nela que se dá sua formação.

A IES tem papel crucial na formação dos futuros contadores, uma vez que "a universidade é o local que tem, entre outras, a função de favorecer uma formação integral em todos os âmbitos" (Bertolin et al., 2013, p. 118). Roth et al. (2013, p. 115) complementam esse pensamento ao afirmar que "a função de uma universidade é formar quadros superiores com capacidade de reflexão crítica e social, propiciando respostas a sociedade através do fornecimento de profissionais para o país". Vendramin, Araujo, Lima, Farias e Gilberto (2015) ressaltam o impacto do mercado de trabalho no ensino superior, dado que uma das funções das IES é a formação do profissional para o mercado de trabalho, logo as IES devem acompanhar as demandas mercadológicas e sempre, que necessário, repensar o profissional que está sendo formado.

Além dos aspectos institucionais e regulatórios é importante ressaltar, ainda, a importância dos fatores humanos envolvidos na formação dos futuros contadores: corpo docente e corpo discente. $\mathrm{O}$ docente, independente da área de conhecimento e atuação, desempenha importantíssimo papel na educação por criar possibilidades para a construção e produção do conhecimento (Freire, 2000).

Já no ensino superior recaem sobre os docentes as expectativas do êxito no processo educacional e, ainda neles, são refletidas as expectativas sobre a formação de profissionais e cidadãos (Nassif \& Hanashiro, 2002), fora o fato de serem os responsáveis pela escolha dos métodos de ensino e avaliação, organizadores de currículo, dentre outras atribuições importantes para a construção e compartilhamento de conhecimento, bem como do desenvolvimento de seus estudantes (Lima, 2018). Especificamente na Contabilidade observa-se uma docência aprendida a partir de modelos a serem seguidos e a serem evitados, um cenário de reprodução de práticas pedagógicas, ingresso na carreira sem formação e preparo, além de sentimentos de insegurança no começo da profissão, mas que se alteram para sentimentos de prazer e satisfação ao decorrer da vida profissional (Araujo, Miranda, \& Pereira, 2017; Ferreira, 2015; Lima \& Araujo, 2019). 
Sobre os estudos relacionados ao corpo discente, justificam-se devido à importância colocada no estudante como protagonista de sua aprendizagem e desenvolvimento principalmente, para os autores, o uso de metodologias ativas e o desenvolvimento da autonomia (Freire, 2000; Santos, 2001; Vendramin, 2018). Além dos estudos relacionados aos estudantes atualmente matriculados, é importante também o acompanhamento dos egressos dos cursos, visto que a partir dessa análise é possível avaliar a qualidade do ensino, as práticas docentes e a realização do PPP (Guimarães et al., 2010).

Diante do cenário apresentado, cabe ressaltar os achados de Vendramin e Araujo (2016), os quais destacam que, no contexto da pesquisa contábil, a área de Educação Contábil encontrase em fase inicial, em processo de construção e solidificação de seus estudos. Tal apontamento se deve a dois indícios: i) número reduzido de doutores em Contabilidade que defenderam teses sobre o tema Ensino Contábil e que seguiram suas carreiras de pesquisa na mesma linha; ii) a trajetória de pesquisa dos orientadores em Ensino Contábil é marcada por intercalar a publicação e orientação em temas do Ensino Contábil com a publicação e orientação em outros temas de pesquisa.

\subsection{Por que pesquisar a Pesquisa Contábil?}

Como discutido anteriormente, a construção do conhecimento é um processo altamente influenciado pelos atores do campo social e pelo contexto histórico, social e econômico vigente. Dessa maneira, é preciso que "como pesquisadores, devemos confrontar continuamente questões da natureza e dos pressupostos do conhecimento que estamos produzindo, para quem o estamos produzindo e por que o estamos produzindo" (Haynes, 2008, p. 543, tradução própria).

Para que tal confrontação seja possível, se faz necessário uma análise prévia das produções existentes. Martins (2012) construiu uma narrativa histórica acerca da pesquisa contábil brasileira e analisou suas principais características. Os resultados do autor apontam para um baixo entendimento dos pesquisadores e pesquisadoras da área acerca dos pressupostos filosóficos do planejamento e execução de uma pesquisa científica, o que pode explicar a baixa diversidade paradigmática ou, como põe o autor, "um cientificismo monoparadigmático desinformado".

Homero Junior (2017) analisa a constituição do campo científico contábil no Brasil visando explicando a ausência de uma linha de pesquisa interpretativa e crítica em Contabilidade. Para tanto, o autor realiza uma revisão da literatura sobre a Contabilidade brasileira e identifica uma baixa autonomia do campo científico em relação ao campo profissional. Homero Junior e Said (2018) demonstram, ainda, que o campo científico contábil no Brasil apresenta características de genderização em relação a algumas temáticas.

Ganz et al. (2019) discutem como a academia brasileira tem cobrado por inovações teóricas, práticas e metodológicas, contudo, tem ao mesmo tempo rejeitado as diversas tentativas de pesquisadores e pesquisadoras não mainstream de fazerem tais contribuições por meio de pesquisas interdisciplinares e com aportes teóricos e metodológicos diversos. Com o avanço e solidificação dos rankings dos periódicos científicos, tem surgido a discussão sobre o impacto das publicações nos processos de contratação e promoção de acadêmicos em diferentes fases da carreira (Bernardi \& Collins, 2019), no processo constitutivo das identidades acadêmicas (Malsch \& Tessier, 2015), na qualidade das pesquisas (Gendron, 2008) e até mesmo no interesse e adesão de pesquisadores e pesquisadoras nas áreas de pesquisa contábil (Tharapos \& Marriott, 2020; Khosa, Burch, Ozdil, \& Wilkin, 2020).

Quanto as redes de autoria, Dias et al. (2020) demonstram que essas, em Contabilidade e Administração, costumam ter como pontos centrais homens em regiões mais privilegiadas do país. Por fim, destaca-se a importância do estudo sobre a pesquisa contábil devido as discussões a respeito da possível estagnação da área (Moser, 2012; Rebele \& St. Pierre, 2015) e da necessidade de reinvenção e inovação em termos teóricos e metodológicos. 


\section{METODOLOGIA}

O presente trabalho tem como objetivo analisar o campo de estudos sobre ensino e pesquisa contábil do USP International Conference in Accounting e do Congresso USP de Iniciação Científica em Contabilidade. A escolha de analisar especificamente os artigos aceitos, apresentados e publicados pelos referidos congressos dá-se pelo fato de que o evento pode ser considerado o principal espaço para debates na área de Controladoria e Contabilidade no Brasil, sendo composto por duas categorias: "Congresso USP de Iniciação Científica em Contabilidade", cujo critério para a participação é a particularidade de que o primeiro autor deve ser aluno de graduação, e o "USP International Conference in Accounting". O congresso tem por objetivo gerar o intercâmbio de estudos e conhecimentos, assim como a exposição de ideias sobre a teoria e a prática da controladoria e da contabilidade, aproximando profissionais dessa área e pesquisadores acadêmicos em momentos como apresentações de trabalhos, workshops, fóruns e palestras.

Em relação aos objetivos, a pesquisa pode ser classificada como exploratória de caráter documental - visto que analisa documentos não interpretados por outrem anteriormente (Silva, 2020). Acerca dos procedimentos para coleta de dados seguimos o proposto por Lima e Mioto (2007), apresentados na Tabela 1.

Tabela 1

\section{Parâmetros da pesquisa Bibliográfica}

\begin{tabular}{cc}
\hline Parâmetro de Lima e Mioto (2007) & Realização Empírica \\
\hline Parâmetro temática & Educação \\
Parâmetro linguístico & Português, inglês e espanhol \\
Parâmetro cronológico & $2001-2020 / 2004-2020$ \\
Principais fontes & Anais dos congressos \\
\hline
\end{tabular}

Fonte: Dados da pesquisa com base em Lima e Mioto (2007).

A obtenção dos dados ocorreu por meio de consulta aos anais dos congressos diretamente no website dos dois eventos. Para o USP International Conference in Accounting, foi considerado o período de 2001 a 2020, abrangendo, assim, todos os anos já realizados do encontro, enquanto para o Congresso USP de Iniciação Científica foi considerado o período de 2004 a 2020, compreendendo todos os anos de sua realização até o momento da execução da pesquisa. Ao total, foram analisados 209 artigos relativos ao International e 126 artigos referente ao Congresso de Iniciação Científica.

Para a análise dos dados foram adotadas técnicas bibliométricas - como análise de publicações por autor, instituição ou tema e número de coautores/colaboradores (Soares, Picolli, \& Casagrande, 2018) - e de estatística descritiva mais a análise de conteúdo. Dessa maneira, após a obtenção dos trabalhos contidos nos anais dos dois congressos, foram tabulados os dados relativos à autoria dos artigos e instituição de vínculo dos autores, título e palavras-chave, além de seu objetivo e metodologia. Visando traçar um cenário macro das pesquisas aceitas e publicadas pelos congressos, os artigos foram classificados em onze eixos: (i) Rendimento Acadêmico; (ii) Currículo; (iii) Docência; (iv) Evasão; (v) História da Contabilidade; (vi) Levantamento Bibliográfico; (vii) Metodologias de Ensino e Aprendizagem; (viii) Corpo Discente; (ix) Perfil Profissional; (x) Pesquisa Científica; (xi) Outros. 


\section{RESULTADOS}

Após o levantamento dos dados, teve início o processo de análise deles. Cabe situar a área de ensino no contingente total de artigos aceitos nos congressos pesquisados nesse levantamento, quais sejam Congresso Internacional e Congresso de Iniciação Científica. A Tabela 2 demonstra que a média de aceitação de artigos na área de Educação Contábil é de $8,11 \%$ para o Congresso Internacional e 15,38\% para o de Iniciação Científica.

Para o Congresso Internacional, o percentual de participação variou entre 1,85\% (2013) até $13,43 \%$ (2018). Já para o Congresso de Iniciação Científica, a participação ficou entre 4,35\% (2017) e 34,15\% (2007). Não é possível observar um comportamento constante entre os anos, seja ele crescente ou decrescente, para ambos os congressos. Esse dado corrobora os achados de Vendramin (2014), que demonstrou uma participação de $15 \%$ de artigos na área de Educação e Pesquisa Contábil nos Congressos USP e Anpcont entre os anos de 2010 e 2013.

Tabela 2

Quantidade de artigos publicados por ano

\begin{tabular}{|c|c|c|c|c|c|c|}
\hline & \multicolumn{3}{|c|}{ Congresso Internacional } & \multicolumn{3}{|c|}{ Iniciação Científica } \\
\hline & $\begin{array}{c}\text { Total Artigos } \\
\text { Aceitos no } \\
\text { Congresso }\end{array}$ & $\begin{array}{c}\text { Artigos } \\
\text { Aceitos Área } \\
\text { Educação }\end{array}$ & $\%$ & $\begin{array}{c}\text { Total Artigos } \\
\text { Aceitos no } \\
\text { Congresso }\end{array}$ & $\begin{array}{c}\text { Artigos } \\
\text { Aceitos Área } \\
\text { Educação }\end{array}$ & $\%$ \\
\hline 2001 & 74 & 7 & 9,46 & - & - & - \\
\hline 2002 & 85 & 7 & 8,24 & - & - & - \\
\hline 2003 & 101 & 11 & 10,89 & - & - & - \\
\hline 2004 & 100 & 5 & 5 & 20 & 2 & 10 \\
\hline 2005 & 100 & 2 & 2 & 20 & 3 & 15 \\
\hline 2006 & 150 & 7 & 4,67 & 32 & 6 & 18,75 \\
\hline 2007 & 120 & 14 & 11,67 & 41 & 14 & 34,15 \\
\hline 2008 & 84 & 10 & 11,9 & 22 & 6 & 27,27 \\
\hline 2009 & 68 & 8 & 11,76 & 17 & 4 & 23,53 \\
\hline 2010 & 101 & 8 & 7,92 & 39 & 6 & 15,38 \\
\hline 2011 & 104 & 10 & 9,62 & 35 & 5 & 14,29 \\
\hline 2012 & 106 & 10 & 9,43 & 49 & 3 & 6,12 \\
\hline 2013 & 54 & 1 & 1,85 & 53 & 10 & 18,87 \\
\hline 2014 & 134 & 9 & 6,72 & 45 & 6 & 13,33 \\
\hline 2015 & 132 & 8 & 6,06 & 57 & 8 & 14,04 \\
\hline 2016 & 112 & 10 & 8,93 & 42 & 6 & 14,29 \\
\hline 2017 & 153 & 4 & 2,61 & 69 & 3 & 4,35 \\
\hline 2018 & 201 & 27 & 13,43 & 89 & 11 & 12,36 \\
\hline 2019 & 196 & 19 & 9,69 & 69 & 15 & 21,74 \\
\hline 2020 & 402 & 32 & 7,96 & 120 & 18 & 15 \\
\hline Total & 2.577 & 209 & 8,11 & 819 & 126 & 15,38 \\
\hline
\end{tabular}

Fonte: Dados da pesquisa.

Observa-se que a área temática de ensino e pesquisa aparenta ter uma média estável de participação nos eventos de âmbito contábil como um todo. Conforme Vendramin e Araújo (2016), os pesquisadores desse campo comumente precisam mesclar suas publicações na área de ensino e pesquisa com publicações em outras áreas da contabilidade, o que pode ser um indicativo de manutenção da média de participação nos eventos científicos, solidificando a 
pesquisa que discute temas ligados ao mainstream contábil. Esse resultado é semelhante ao contexto internacional em que publicações em periódicos de alto impacto influenciam processos de contratação e promoção, sendo que os periódicos de nicho - tais como educação, história e sustentabilidade - tendem a ter notas mais baixas e atrair menos pesquisadores e pesquisadoras (Tharapos \& Marriot, 2020).

Além das dinâmicas entre o que é aceito ou não por determinada comunidade, para analisar um campo científico é preciso analisar o grupo que o compõe e como esse se organiza. Considerando que a ciência moderna tem se pautado cada vez mais no estabelecimento de parcerias e redes de coautoria (Rey-Rocha, Martín-Sempere, \& Garzón-García, 2002), apresentamos na Tabela 3 a distribuição do número de autores por artigo nos congressos analisados.

Tabela 3

\section{Distribuição de Autores}

\begin{tabular}{ccccccc}
\hline $\begin{array}{c}\text { Número de Autores } \\
\text { por Artigo }\end{array}$ & $\begin{array}{c}\text { Congresso } \\
\text { Internacional }\end{array}$ & $\boldsymbol{\%}$ & Congresso de IC & $\boldsymbol{\%}$ & Total & $\%$ \\
\hline $\mathbf{1}$ & 16 & 7,66 & 2 & 1,59 & 18 & 5,37 \\
$\mathbf{2}$ & 67 & 32,06 & 32 & 25,40 & 99 & 29,55 \\
$\mathbf{3}$ & 49 & 23,44 & 36 & 28,57 & 85 & 25,37 \\
$\mathbf{4}$ & 54 & 25,84 & 32 & 25,40 & 86 & 25,67 \\
$\mathbf{5}$ & 2 & 0,96 & 7 & 5,56 & 9 & 2,26 \\
$\mathbf{6}$ & 0 & 0 & 2 & 1,59 & 2 & 0,60 \\
$\begin{array}{c}\text { Autoria não } \\
\text { identificada }\end{array}$ & 21 & 10,05 & 15 & 11,90 & 36 & 10,75 \\
\hline Total & $\mathbf{2 0 9}$ & $\mathbf{1 0 0}$ & $\mathbf{1 2 6}$ & $\mathbf{1 0 0}$ & $\mathbf{3 3 5}$ & $\mathbf{1 0 0}$ \\
\hline
\end{tabular}

Fonte: Dados da pesquisa.

O cenário apresentado na Tabela 3 destaca a distribuição de publicações dos dois congressos analisados a partir da quantidade de autores por artigo. Observa-se que a quase totalidade dos textos de autoria única encontra-se no USP International Conference in Accounting, enquanto a dos artigos que envolvem cinco e seis autores alocam-se no congresso de Iniciação Científica. Infere-se que os pesquisadores do congresso de Iniciação Científica, que obrigatoriamente devem ser vinculados à graduação, são inseridos nos projetos de pesquisa de seus orientadores. A partir do quantitativo total referente aos dois eventos, a somatória dos artigos com 2,3 e 4 autores representa $80,59 \%$, demonstrando que as publicações não são isoladas e ocorrem mediante parcerias.

Dias et al. (2020) destacam ainda que, ao analisar as redes de autoria, é importante considerar fatores sociais tais como o sexo dos autores e autoras. Diante disso, na Tabela 4 são apresentados os quantitativos da participação de homens e mulheres em ambos os congressos.

Tabela 4

\section{Quantidade de Homens e Mulheres}

\begin{tabular}{ccccccc}
\hline & Congresso Internacional & $\boldsymbol{\%}$ & Congresso de IC & $\boldsymbol{\%}$ & Total & \% \\
\hline Homens & 286 & 54,79 & 152 & 43,18 & 438 & 50,11 \\
Mulheres & 236 & 45,21 & 200 & 56,82 & 436 & 49,89 \\
\hline Total de Autores & $\mathbf{5 2 2}$ & $\mathbf{1 0 0}$ & $\mathbf{3 5 2}$ & $\mathbf{1 0 0}$ & $\mathbf{8 7 4}$ & $\mathbf{1 0 0}$ \\
\hline
\end{tabular}

Fonte: Dados da pesquisa.

Nota. Não foi considerada unicidade de autoria, assim, caso a pessoa tenha três artigos em determinado ano, será contada três vezes na Tabela 4. 
Com relação à distribuição de autoria entre homens e mulheres, quando observados os dois congressos de forma conjunta, existe um equilíbrio. Entretanto, ao analisar individualmente cada congresso, é possível perceber uma maioria de autores homens no congresso Internacional $(54,79 \%)$ e uma maioria de autoras mulheres no congresso de Iniciação Científica $(56,82 \%)$. Nota-se ainda que existe uma concentração de autores e autoras no congresso Internacional, representando quase $60 \%$ da amostra do presente estudo. Essa concentração pode ser reflexo de uma maior quantidade de artigos em tal evento.

Homero Junior e Said (2018) argumentam que áreas como sustentabilidade e educação apresentam uma predominância de mulheres, contudo menor prestígio na área. Os resultados do presente estudo reforçam essa ideia ao mostrar que no congresso de maior influência - o Internacional - os homens detêm maior participação, enquanto no congresso de menor prestígio, existe uma maior participação feminina. Essa presença de homens em espaços e áreas tidas como socialmente femininas, como a educação, é explicada pelo fenômeno glass escalator que demonstra como homens possuem maior facilidade de ascensão e obtenção de prestígio em áreas "femininas" (Williams, 1992).

A maior presença de mulheres no congresso de iniciação científica ainda pode ser explicada pelo fato de esse ser voltado para a publicação de resultados de pesquisas de estudantes de graduação e, como mostra Casa Nova (2019), as professoras titulares em Contabilidade consideram o processo de orientação - e não a publicação - de trabalhos científicos como um indicador de sucesso. A diferença de participação entre homens e mulheres nos dois eventos também pode ser explicada pela formação de redes de autoria que, como demonstra o trabalho de Dias et al. (2020), costumam ser centralizadas por homens na área de Contabilidade e Administração.

Tabela 5

Ranking das IES de vinculação dos autores que publicaram no Congresso Internacional

\begin{tabular}{ccc}
\hline Instituição de Ensino Superior (IES) & Ocorrências & \% \\
\hline Universidade de São Paulo & 95 & 18,20 \\
Universidade Regional de Blumenau & 40 & 7,66 \\
Universidade Federal de Uberlândia & 38 & 7,28 \\
Universidade Federal do Paraná & 33 & 6,32 \\
Universidade Federal de Minas Gerais & 33 & 6,32 \\
Universidade Federal do Rio Grande do Norte & 32 & 6,13 \\
Universidade Federal do Rio Grande & 22 & 4,21 \\
Universidade Estadual do Oeste Do Paraná & 21 & 4,02 \\
Universidade Federal de Santa Catarina & 18 & 3,45 \\
Universidade Federal de Santa Maria & 14 & 2,68 \\
\hline Total & $\mathbf{3 4 6}$ & 66,28 \\
\hline
\end{tabular}

Fonte: Dados da pesquisa.

Considerando as 10 Instituições de Ensino Superior que mais publicaram artigos no USP International Conference in Accounting, corroborando com as expectativas, a Universidade de São Paulo lidera o ranking. As principais razões para esse achado são: o pioneirismo relativo ao curso de Ciências Contábeis no Brasil, o fato da IES ser sede do evento e o fato de ser a única que possui linha de pesquisa específica de Educação e Pesquisa em Contabilidade. Salienta-se que, entre as 10 ranqueadas, apenas uma universidade encontra-se fora do eixo Sul-Sudeste (Universidade Federal do Rio Grande do Norte), confirmando os resultados de Dias et al. (2020) que demonstram como regiões mais sociais e economicamente privilegiadas centralizam as redes de pesquisa e autoria. 
É importante ainda ressaltar que os dados relativos às IES podem estar enviesados por aquelas que possuem programas de pós-graduação - visto que seis das dez melhor ranqueadas possuem curso de doutorado - pois, tais programas, cobram publicações periódicas de seus discentes (Altoé, Fragalli, \& Espejo, 2014).

Tabela 6

Ranking das IES de vinculação dos autores que publicaram no Congresso de Iniciação Científica

\begin{tabular}{ccc}
\hline Instituição de Ensino Superior (IES) & Ocorrências & \% \\
\hline Universidade Federal de Pernambuco & 28 & 7,95 \\
Universidade Federal de Uberlândia & 27 & 7,67 \\
Universidade Federal de Santa Catarina & 27 & 7,67 \\
Universidade Estadual de Montes Claros & 22 & 6,25 \\
Universidade Federal da Paraíba & 19 & 5,40 \\
Universidade de São Paulo & 17 & 4,83 \\
Universidade Federal do Rio Grande do Norte & 13 & 3,69 \\
Universidade Católica de Brasília & 13 & 3,69 \\
Universidade Estadual de Feira de Santana & 12 & 3,41 \\
Universidade Federal de Minas Gerais & 10 & 2,84 \\
\hline Total & $\mathbf{1 8 8}$ & 53,40 \\
\hline
\end{tabular}

Fonte: Dados da pesquisa.

Contrariando as expectativas, a USP encontra-se apenas em sexto lugar no ranking de IES que mais publicaram no Congresso de Iniciação Científica. Ressalta-se a expressiva participação de universidades localizadas na Região Nordeste, representando $25 \%$ das dez primeiras, sinalizando que os orientadores incentivam seus alunos de graduação a participarem da área de Educação no evento. As universidades que figuram em ambas as listas são: Universidade de São Paulo, Universidade Federal de Minas Gerais, Universidade Federal de Uberlândia e Universidade Federal do Rio Grande do Norte.

Considerando a importância dos autores envolvidos no processo de construção do conhecimento, considera-se que a análise individual de autoria e persistência dos pesquisadores no campo científico é essencial. Na Tabela 7 são apresentados os dados referentes a quantidade de artigos por autor/autora em cada um dos congressos no período analisado.

Tabela 7

Quantidade de artigos por autor

\begin{tabular}{ccc}
\hline & Congresso Internacional & $\begin{array}{c}\text { Congresso de Iniciação } \\
\text { Científica }\end{array}$ \\
\hline 1 Artigo & 304 & 252 \\
2 Artigos & 51 & 28 \\
3 Artigos & 13 & 10 \\
4 Artigos & 5 & 1 \\
5 Artigos & 4 & 2 \\
6 Artigos & 2 & 0 \\
7 Artigos & 0 & 0 \\
8 Artigos & 1 & 0 \\
9 Artigos & 2 & 0 \\
\hline
\end{tabular}

Fonte: Dados da pesquisa. 
O cenário observado na Tabela 8 é caracterizado por um alto número de autores onetimers, ou seja, que publicaram apenas um artigo no período analisado. Tal resultado é semelhante a conjuntura da pesquisa em Contabilidade Gerencial nos periódicos contábeis de 2008 a 2012, em que existiam 623 autores one-timer (Souza et al., 2016).

Além da predominância de one-timers, na linha de maneira geral, é possível observar também que os pesquisadores e pesquisadoras da área costumam diversificar as temáticas estudadas dentro dessa repartição. A conclusão é similar à de Vendramin (2014) e pode indicar que os pesquisadores não desejam aprofundar os estudos acerca das temáticas, o que explicaria a possível estagnação da pesquisa em Contabilidade de maneira geral e em Educação Contábil (Rebele \& St. Pierre, 2015). Existe também a possibilidade de os pesquisadores e pesquisadoras da área manterem a linha de ensino e pesquisa como uma linha de dedicação secundária e publiquem em outras linhas mais aceitas por congressos, periódicos e pela área de maneira geral. Tal fato pode ser explicado pela existência formal da linha de pesquisa em ensino contábil somente no programa de pós-graduação da FEA/USP.

Acerca de quem são os autores/autoras mais prolíficos na área, observa-se na Tabela 8 a indicação de seus nomes e o total de artigos nesse período. Para organizar os dados da Tabela 8, estabeleceu-se que seriam incluídos os cinco autores/autoras mais prolíficos de cada congresso.

Tabela 8

Autores mais Prolíficos nos Congressos

\begin{tabular}{cccc}
\hline Internacional & \multicolumn{2}{c}{ Iniciação Científica } \\
\hline Autor(a) & Total de Artigos & Autor(a) & Total de Artigos \\
\hline Gilberto De Andrade Martins & 9 & Carlos Renato Theóphilo & 5 \\
Jacqueline Veneroso Alves Da Cunha & 9 & Idalberto José Das Neves Júnior & 5 \\
Gilberto José Miranda & 8 & Edmery Tavares Barbosa & 4 \\
Edgard Bruno Cornacchione Junior & 6 & 10 autores/autoras & 3 \\
Edvalda Araújo Leal & 6 & 28 autores/autoras & 2 \\
\hline
\end{tabular}

Fonte: Dados da pesquisa.

Observa-se que, no congresso de iniciação científica, existe uma grande concentração entre autores/autoras com três e dois artigos, tornando inviável elencar os cinco autores/autoras mais prolíficos, ao contrário do congresso internacional. O resultado da Tabela 8 reforça o argumento de Vendramin (2014) ao indicar que, embora o(a) pesquisador(a) tenha familiaridade com os temas da linha de ensino contábil, ele(a) precisa desenvolver pesquisas em outras linhas, mantendo o ensino contábil como sua linha secundária.

Tabela 9

Eixos mais pesquisados por congresso

\begin{tabular}{cccc}
\hline Eixos & Internacional & Iniciação & Total \\
\hline Corpo Discente & 44 & 24 & 68 \\
Metodologias de Ensino e Aprendizagem & 27 & 17 & 44 \\
Rendimento Acadêmico & 24 & 18 & 42 \\
Levantamento Bibliográfico & 27 & 13 & 40 \\
Perfil Profissional & 22 & 15 & 37 \\
Currículo & 15 & 12 & 27 \\
Docência & 16 & 8 & 24 \\
Pesquisa Científica & 12 & 6 & 18 \\
Outros & 10 & 3 & 11 \\
Evasão & 6 & 5 & 11 \\
\hline História da Contabilidade & 6 & 5 & $\mathbf{3 3 5}$ \\
\hline TOTAL & $\mathbf{2 0 9}$ & $\mathbf{1 2 6}$ & \\
\hline
\end{tabular}

Fonte: Dados da pesquisa. 
Acerca dos temas mais pesquisados, observa-se na Tabela 9 a predominância dos temas relacionados ao corpo discente, às metodologias de ensino e aprendizagem e do rendimento acadêmico. Repara-se ainda a grande presença de levantamentos bibliográficos que são submetidos à essa linha, mas que podem dizer respeito às outras áreas de conhecimento de contabilidade. É importante notar a presença do tema de "História da Contabilidade" que, apesar de constituir uma linha de pesquisa própria, acaba sendo encaixado nas outras temáticas do congresso e nota-se também o fato de que pesquisas acerca da própria prática de pesquisa acumulam apenas 18 artigos no período analisado, ou seja, cerca de 5\%.

O cenário apresentado pela Tabela 9 destaca pontos de convergência e divergência com a literatura internacional. Por exemplo, Apostolou, Dorminey, Hassell e Hickey (2019) e Apostolou, Dorminey e Hassell (2020) demonstram que, nos principais periódicos internacionais de educação contábil, a pesquisa empírica tem dado cada vez mais espaço a materiais didáticos e casos de ensino. Ao mesmo tempo, mostra que áreas de nicho como história e epistemologia se mantém à margem - mesmo dentro do campo de ensino e pesquisa (Tharapos \& Marriott, 2020).

Ao tratar do Ensino Contábil, entendemos que o assunto é mais amplo do que apenas olhar para as questões que envolvem os discentes. É necessária a elaboração de pesquisas que olhem também para o corpo docente e para as instituições de ensino. Assim, os temas possíveis na área são inúmeros, como, por exemplo, formação e identidade docente, orientações, normatizações dos cursos, da profissão contábil e da profissão docente, entre inúmeras outras possibilidades. Dessa forma, espera-se que um maior envolvimento de pesquisadores com os temas de ensino contábil possa contribuir de forma significativa com o desenvolvimento total da linha, tendo em vista a possibilidade de pesquisas nos outros tópicos até então pouco explorados.

\section{CONSIDERAÇÕES FINAIS}

Com vistas a cumprir o objetivo de analisar a produção científica sobre Ensino e Pesquisa Contábil do USP International Conference in Accounting e do Congresso USP de Iniciação Científica em Contabilidade, foi realizado um levantamento bibliográfico cujos dados foram obtidos nos anais dos referidos eventos, especificamente entre os anos 2001 a 2020 para o Congresso Internacional e 2004 a 2020 para o Congresso de Iniciação Científica.

Os dados apontam uma baixa participação de artigos na área de Educação no total analisado dos artigos do USP International Conference in Accounting, média que melhora um pouco quando se fala do Congresso de Iniciação Científica. No que tange à quantidade de autores, o USP International Conference in Accounting tende a ter menos coautoria em cada artigo, situação que se inverte no Congresso de Iniciação Científica, com um percentual maior de cinco ou seis autores. Entretanto, quando o olhar se volta para o somatório total referente aos dois eventos, a somatória dos artigos com dois, três e quatro autores representa 80,6\%, demonstrando que as publicações não são isoladas e ocorrem mediante parcerias.

$\mathrm{O}$ item analisado relativo ao sexo dos autores demonstrou uma predominância de mulheres no Congresso de Iniciação Científica e de homens no USP International Conference in Accounting. Esse resultado vincula-se ao conceito de glass escalator ao demonstrar maior participação de mulheres em áreas pré-conceituadas como sendo de menor prestígio (Williams, 1992), ao fato de as mulheres considerarem o processo de orientação como um indicativo de sucesso acadêmico (Casa Nova, 2019) e, ainda, ao fato de o Congresso de Iniciação Científica estar voltado aos trabalhos fruto de Iniciações Científicas desenvolvidas nos cursos de graduação.

Sobre as Instituições de vinculação informadas pelos autores, a Universidade de São Paulo tem destaque no USP International Conference in Accounting, perfazendo um total de artigos superior ao dobro da segunda IES. Já no Congresso de Iniciação Científica, os números de ocorrências por IES são bastante próximos, de forma que não se pode afirmar que existe uma 
liderança, entretanto os destaques ficam com a Universidade Federal de Pernambuco, Universidade Federal de Uberlândia e Universidade Federal de Santa Catarina.

Ao trazer os dados dos autores que mais aparecem nos artigos aceitos, observou-se que no USP International Conference in Accounting apenas um autor possui 9 artigos, dois possuem 6 artigos, um possui 5 artigos, cinco possuem 4 artigos e doze possuem 3 artigos, nosso ponto de corte para análise. Entretanto, os autores apresentaram uma grande variedade com relação aos temas que são tratados em seus textos, não existindo uma linha de pesquisa dentro da área de Educação Contábil. Já no Congresso de Iniciação Científica, a maior quantidade de artigos de um mesmo autor chegou a 5 textos, também pulverizados entre os temas dentro da Educação Contábil.

Acerca das temáticas, observa-se a predominância dos temas relacionados à educação, principalmente os que tratam o corpo discente, metodologias de ensino e aprendizagem e rendimento acadêmico. Dessa maneira, observa-se a necessidade de estudos sobre métodos de pesquisa, assim como de reflexões epistemológicas e ontológicas, além das implicações decorrentes da adoção de índices de qualidade que incentivam a produtividade. Destaca-se, ainda, que dentre as pesquisas relacionadas ao ensino existe a carência de mais trabalhos no tocante à evasão dos estudantes e de artigos acerca da gestão das IES. Diante do contexto da pandemia, observa-se uma possível agenda de pesquisa para analisar esse contexto de exceção que reconfigura o cenário educacional.

Com limitações, se realça o fato de não existir dados comparativos às demais áreas do congresso e a presença de dados faltantes por não estarem disponíveis nos anais dos congressos. Outra limitação é o fato de o trabalho não aprofundar a discussão das relações entre autoras e autores da área. Para pesquisas futuras, é recomendada a análise qualitativa sobre a constituição do campo científico de ensino e pesquisa, a partir de entrevistas com os principais agentes identificados nesse artigo. Sugere-se também a análise das redes de autoria que se pautem nos principais centros e agentes do campo como feito por Dias et al. (2020). Por fim, é recomendada a análise dos principais métodos e metodologias dos artigos e as posições ontológicas e epistemológicas da área.

\section{REFERÊNCIAS}

Altoé, S. M. L., Fragalli, A. C., \& Espejo, M. M. D. S. B. (2014). A "dor do crescimento": um estudo sobre o nível de estresse em pós-graduandos de contabilidade. Revista Gestão Universitária na América Latina-GUAL, 7(1), 213-233.

Apostolou, B., Dorminey, J. W., Hassell, J. M., \& Rebele, J. E. (2018). Accounting education literature review (2017). Journal of accounting education, 43, 1-23.

Apostolou, B., Dorminey, J. W., Hassell, J. M., \& Hickey, A. (2019). Accounting education literature review (2018). Journal of Accounting Education, 47, 1-27.

Apostolou, B., Dorminey, J. W., \& Hassell, J. M. (2020). Accounting education literature review (2019). Journal of Accounting Education, 51, 100670.

Araújo, T. S., Miranda, G. J., \& Pereira, J. M. (2017). Satisfaction among accounting professors in Brazil. Revista Contabilidade \& Finanças, 28(74), 264-281.

Bernardi, R. A., \& Collins, K. Z. (2019). "Leveling the playing field" when ranking accountingeducation authors. Journal of Accounting Education, 46, 1-25. 
Bertolin, R. V., Souza, R. F., Monteiro Neto, D., Pedezzi, L. S., Cambi, B., Pierson, A. H. C., ... Zuin, V. G. (2013). A Integração PIBID/UFSCar-Escola de Educação Básica como um espaço de construção de uma postura interdisciplinar. In M. C. Sousa, \& C. M. P. Marques (Orgs.). Formação inicial de professores: Parceria universidade-escola na formação de licenciados (pp. 117-136). Curitiba: Appris.

Bourdieu, P. (1983). O campo científico. In R. Ortiz (Org.). Pierre Bourdieu: Sociologia (v. 39, pp. 122-155). São Paulo: Ática.

Burrell, B., \& Morgan, G. (1979). Sociological paradigms and organisational analysis: Elements of the sociology of corporate life. Burlington, Vermont: Ashgate Publishing Company.

Caniato, A. M. P. (2005). Resenha: a questão dos paradigmas na psicologia. Psicologia \& Sociedade, 17(3), 82-84.

Casa Nova, S. P. C. (2019). Ridin' down the highway: Reflections on the trajectories of female professors in academia. Annals of Qualitative Research and Critical Accounting: A Latin American Conference - QRCA, Bogotá, Colômbia, 2.

Chua, W. F. (1986). Radical developments in accounting thought. The Accounting review, 61(4), 601-632.

Costa, F., \& Martins, G. A. (2017). Um olhar Bourdieusiano sobre as estruturas sociais do campo científico contábil brasileiro. Revista Universo Contábil, 13(4), 8-32.

Cruz, M. O., Miranda, G. J., \& Leal, E. A. (2020). As metodologias de ensino ativam o desenvolvimento de habilidades profissionais? Revista Contemporânea de Contabilidade, 17(45).

Dias, A., Ruthes, S., Lima, L., Campra, E., Silva, M., Bragança de Sousa, M., \& Porto, G. (2020). Network centrality analysis in management and accounting sciences, RAUSP Management Journal, 54(4).

Ferreira, M. M. (2015). Docência no ensino superior: Aprendendo a ser professor de contabilidade (Tese de Doutorado). Universidade Federal de São Carlos - UFSCar, São Carlos, SP, Brasil.

Fogarty, T. J., \& Liao, C. H. (2009). Blessed are the gatekeepers: A longitudinal study of the editorial boards of The Accounting Review. Issues in Accounting Education, 24(3), 299-318.

Freire, P. (2000). Pedagogia da autonomia: Saberes necessários à prática educativa (15a ed.). São Paulo: Editora Paz e Terra.

Ganz, A. C. S., Lima, J. P. R., \& Haveroth, J. (2019). Velhos problemas, novos olhares: Etnografia sobre a experiência de futuros doutores em contabilidade. Anais do USP International Conference in Accounting, São Paulo, SP, Brasil, 19.

Gendron, Y. (2008). Constituting the academic performer: the spectre of superficiality and stagnation in academia. European Accounting Review, 17(1), 97-127. 
Guimarães, I. P., Slomski, V. G., \& Gomes, S. M. S. (2010). Gestão do projeto políticopedagógico do curso de ciências contábeis e o currículo como instrumento de sua concretização. Revista de Contabilidade da UFBA, 4(1), 36-55.

Haynes, K. (2008). Moving the gender agenda or stirring chicken's entrails? Where next for feminist methodologies in accounting? Accounting, Auditing \& Accountability Journal, 21(4), 539-555.

Homero Junior, P. F. (2017). A constituição do campo científico e a baixa diversidade da pesquisa contábil brasileira. Revista de Educação e Pesquisa em Contabilidade REPeC, 11(3), 307-321.

Homero Junior, P. F., \& Said, R. M. (2018). Genderização da pesquisa contábil brasileira. Anais do USP International Conference in Accounting, São Paulo, SP, Brasil, 18.

Iudícibus, S. (2004). Teoria da contabilidade (7a ed.). São Paulo: Atlas.

Kuhn, T. (1978). A estrutura das revoluções científicas (2a ed.). São Paulo: Perspectiva.

Laffin, M. (2002). Ensino de contabilidade: Componentes e desafios. Contabilidade Vista \& Revista, 13(3), 9-20.

Latour, B. (1987). Science in action. Cambridge, Massachusetts: Harvard University Press.

Lima, J. P. R. (2018). Ser professor: Um estudo da identidade docente na área de ciências contábeis (Dissertação de Mestrado). Universidade de São Paulo - USP, Ribeirão Preto, SP, Brasil.

Lima, J. P. R., \& Araujo, A. M. P. (2019). Tornando-se professor: análise do processo de construção da identidade docente dos professores de contabilidade. Advances in Scientific and Applied Accounting, 1(2), 059-080.

Lima, R. S., Serrano, A. L. M., \& Ferreira, L. O. G. (2020). Perspectiva Kuhniana sobre a Ciência Contábil: do surgimento do paradigma ao período de crise. Revista Catarinense da Ciência Contábil, 19.

Lima, T. C. S. D., \& Mioto, R. C. T. (2007). Procedimentos metodológicos na construção do conhecimento científico: A pesquisa bibliográfica. Revista Katálysis, 10(SPE), 37-45.

Khosa, A., Burch, S., Ozdil, E., \& Wilkin, C. (2020). Current issues in PhD supervision of accounting and finance students: Evidence from Australia and New Zealand. The British Accounting Review, 52(5), 100874. https://doi.org/10.1016/j.bar.2019.100874

Malsch, B., \& Tessier, S. (2015). Journal ranking effects on junior academics: Identity fragmentation and politicization. Critical Perspectives on Accounting, 26, 84-98.

Martins, E. A. (2012). Pesquisa contábil brasileira: Uma análise filosófica (Tese de Doutorado), Universidade de São Paulo - USP, São Paulo, SP, Brasil. 
Martins, E. A., \& Carvalho, L. N. G. (2011). Ciência da contabilidade: Ensaio teórico sobre seu objetivo e objeto. Anais do Congresso USP de Controladoria e Contabilidade, São Paulo, SP, Brasil, 11.

Martins, O. S., \& Monte, P. A. (2009). Mestres em ciências contábeis: Uma análise sob a ótica da teoria do capital humano. Revista de Educação e Pesquisa em Contabilidade, 3(2), 1-22.

Menafra, R. P. (2007). Notas sobre violência epistêmica, vigilância epistemológica e alternativas à produção do conhecimento em ciências sociais. Latitude, 1(2), 66-79.

Miranda, G. J., Santos, L. A. A., Casa Nova, S. P. C., \& Cornacchione Junior, E. B. (2013) A pesquisa em educação contábil: Produção científica e preferências de doutores no período de 2005 a 2009. Revista de Contabilidade \& Finanças, 24(61), 75-88.

Morgan, G. (1980). Paradigms, metaphors, and puzzle solving in organization theory. Administrative Science Quarterly, 25(4), 605-622.

Moser, D. V. (2012). Is accounting research stagnant? Accounting Horizons, 26(4), 845-850.

Nagib, L. D. R. C., \& Silva, D. M. D. (2020). Adoção de metodologias ativas e sua relação com o ciclo de vida e a qualificação docente no ensino de graduação em ciências contábeis. Revista Contabilidade \& Finanças, 31(82), 145-164.

Nassif, V. M. J., \& Hanashiro, D. M. M. (2002). A competitividade das universidades particulares à luz de uma visão baseada em recursos. Revista de Administração Mackenzie, $3(1), 95-114$.

Oliveira, M. C. (2002). Análise dos periódicos brasileiros de contabilidade. Revista Contabilidade \& Finanças, 13(29), 68-86.

Paulo, E., \& Martins, E. (2007). Análise da qualidade das informações contábeis nas companhias abertas. Anais do Encontro da Associação Nacional de Pós-Graduação e Pesquisa em Administração - EnANPAD, Rio de Janeiro, RJ, Brasil, 31.

Peleias, I. R., Silva, G. P. D., Segreti, J. B., \& Chirotto, A. R. (2007). Evolução do ensino da contabilidade no Brasil: Uma análise histórica. Revista Contabilidade \& Finanças, 18(SPE), 19-32.

Rebele, J. E., \& St. Pierre, E. K. (2015). Stagnation in accounting education research. Journal of Accounting Education, 33(2), 128-137.

Rey-Rocha, J., Martín-Sempere, M., \& Garzón-García, B. (2002). Research productivity of scientists in consolidated vs non-consolidated teams: The case of Spanish university geologists. Scientometrics, 55(1), 137-156.

Riccio, E. L., \& Sakata, M. C. G. (2004). Evidências da globalização na educação contábil: Estudo das grades curriculares dos cursos de graduação em universidades brasileiras e portuguesas. Revista de Contabilidade \& Finanças, 15(35), 35-44. 
Roth, L., Espíndola, A. M. S., Santos, V. O., Dorion, E. C. H., Fachinelli, A. C., \& Severo, E. A. (2013). A estrutura do ensino superior no Brasil. Revista Gestão Universitária na América Latina, 6(3), 111-126.

Santos, S. C. (2001). O processo de ensino-aprendizagem e a relação professor-aluno: Aplicação dos "sete princípios para a boa prática na educação de ensino superior". Cadernos de Pesquisa em Administração, 8(1), 69-82.

Sauerbronn, F. F., Ayres, R. M., \& Lourenço, R. L. (2017). Perspectivas pós-coloniais e decoloniais: Uma proposta de agenda de pesquisa em contabilidade no Brasil. Custos $e$ @ gronegócios online, 13(3), 120-148.

Soares, S. V., Lima Filho, R. N., \& Nova, S. P. D. C. C. (2020). Google Acadêmico: uma opção para análise de citações dos periódicos brasileiros de contabilidade?. Revista Gestão Universitária na América Latina-GUAL, 13(2), 140-160.

Soares, S. V., Bulaon, C., Nova, S. P. D. C. C., \& Picolli, I. R. A. (2019). Aprendizagem baseada em problemas para os cursos de ciências contábeis: Desafios e oportunidades de sua adoção. Contextus: Revista Contemporânea de economia e gestão, 17(1), 65-97.

Soares, S., Picolli, I., \& Casagrande, J. (2018). Pesquisa Bibliográfica, Pesquisa Bibliométrica, Artigo de Revisão e Ensaio Teórico em Administração e Contabilidade. Administração: Ensino e Pesquisa, 19(2), 308-339. doi: https://doi.org/10.13058/raep.2018.v19n2.970

Souza, A. A., Avelar, E. A., Boina, T. M., Ribeiro, L. M. P., Santos, T. S., \& Rabelo, J. S. (2016). Análise bibliométrica das pesquisas brasileiras em contabilidade gerencial publicadas entre os anos de 2008 e 2012. Revista de Estudos Contábeis, 7(12), 57-79.

Stroeher, A. M., \& Freitas, H. (2008). O uso das informações contábeis na tomada de decisões em pequenas empresas. Revista RAUSP-e, 1(1).

Tharapos, M., \& Marriott, N. (2020). Beauty is in the eye of the beholder: Research quality in accounting education. The British Accounting Review, 52(5), 100934.

Vendramin, E. (2014). Uma contribuição ao entendimento da formação da linha de pesquisa na área do ensino contábil no Brasil (Dissertação de Mestrado). Universidade de São Paulo USP, Ribeirão Preto, SP, Brasil.

Vendramin, E. O. (2018). Criando caso: Análise do método do caso como estratégia pedagógica no ensino superior da contabilidade (Tese de Doutorado). Universidade de São Paulo - USP, Ribeirão Preto, SP, Brasil.

Vendramin, E. O., \& Araujo, A. M. P. (2016). Contribuição ao entendimento da formação da linha de pesquisa na área de ensino contábil no Brasil. Revista Universo Contábil, 12(1), 6686.

Vendramin, E. O., Araujo, A. M. P., Lima, J. P. R., Farias, R. S., \& Gilberto, T. M. J. (2015). Metodologias ativas de aprendizagem: Um estudo de caso sobre a aplicação do PBL no curso de ciências contábeis. Anais do Encontro de Ensino e Pesquisa em Administração e Contabilidade - EnEPQ, Salvador, BA, Brasil, 5. 
Williams, C. L. (1992). The glass escalator: Hidden advantages for men in the "female" professions. Social problems, 39(3), 253-267. 